\title{
El estado de la tutoría en la formación académica universitaria
}

\section{The state of the tutoring in the formation academic university}

Gabith M. Quispe F.1 \& Otto E. Arellano C.1

${ }^{1}$ Facultad de Ciencias Políticas y Administrativas de la Universidad Nacional de Chimborazo de Ecuador.

Email: gabithmiriam@gmail.com - gabithq@yahoo.es

\section{RESUMEN}

El estudio de la tutoría tiene su base en los estudios sobre la educación y sus implicaciones en el desarrollo económico de un país y es un tema explorado y en continuo progreso. El objetivo de la investigación es determinar si la tutoría es uno de los elementos determinantes para lograr la calidad en el proceso de enseñanza y aprendizaje en el sistema de la educación superior. Se aplica el método deductivo y enfoque cualitativo - descriptivo. Se realiza una revisión bibliográfica relacionada a la tutoría. Se estudia el caso de la Universidad Boliviana - Universidad Técnica de Oruro, Carrera de Administración de Empresas como caso empírico, en ella se aplica un cuestionario a la planta docente, método que permite comparar factores con casos de universidades del Ecuador, Argentina y México. Los resultados demuestran: que la definición de tutoría está asociada a las acciones y funciones del docente tutor en aula; el modelo de tutoría está relacionado con los modelos académicos de la universidad y del sistema de educación superior; los factores medibles de la tutoría son importantes para la calidad educativa en la educación superior universitaria; y la tutoría contribuye en la reducción de la deserción y repitencia principalmente.

\section{ABSTRACT}

The study of mentoring is based on studies on education and its implications for economic development of a country and is a topic explored and continuous progress. The objective of the research is to determine whether mentoring is one of the key elements to achieve quality in the process of teaching and learning in the higher education system. descriptive - qualitative method and deductive approach is applied. A literature review related to tutoring is done. Technical University of Oruro, Carrera Business Administration as empirical case it a questionnaire to the teaching staff, method for comparing factors with cases of universities Ecuador, Argentina and Mexico applies - the case of the Bolivian University is studied. The results show: that the definition of mentoring is associated with actions and functions classroom teacher tutor; mentoring model is related to academic models of the university and higher education system; measurable factors mentoring are important to educational quality in university education; and mentoring contributes to reducing dropout and repetition primarily.

Palabras clave | Key words:

docencia, modelos, rol tutorial, sistema educativo

teaching, models, tutorial role, educational system 


\section{INTRODUCCIÓN}

En América Latina y el mundo el estudio de la tutoría es abordado en estudios sobre la educación, sus implicaciones en el proceso de enseñanza aprendizaje y este en el desarrollo económico de un país es un tema explorado y en continuo progreso. Su aplicación y la teoría está constantemente en construcción, en ese ámbito los estudios están relacionados principalmente buscando relaciones entre la "inversión en el sector educativo y el crecimiento económico, análisis sobre factores asociados a la cobertura y a la educación, la demanda educativa versus rendimiento, financiación y acceso a la educación, gasto público, egresados y satisfacciones" (Cano G. C.A., 2008). Sin embargo, no deja de ser importante el estudio de la tutoría, ya que se constituye en un factor importante para lograr la calidad educativa por su relación en el proceso de enseñanza- aprendizaje. Puesto que la tutoría a pesar de ser considera teóricamente incuestionable en su definición como menciona Lara G.B. (2009) permite responder a cuestionamientos sobre el significado de la tutoría, desde el punto de vista del acompañamiento y sus implicaciones relacionados principalmente con "la calidad académica y la eficiencia de la educación superior en temas como la atención a la reprobación y la prevención de la deserción del alumnado" (Lara et.al., 2009). En ese sentido, la educación universitaria está relacionada con la economía educativa (desde el punto de vista económico e institucional) ya que la educación juega un papel importante para el desarrollo humano y económico de un país, región - localidad, lo que significa que la formación de los estudiantes en la educación superior universitaria responde a la teoría económica que señala que la riqueza de un país depende más de sus recursos humanos que de los naturales, es decir a mayor educación mayor renta, lo que trae consigo que la tutoría se convierta en un elemento importante para lograr la calidad educativa a partir de su incidencia en la reducción de las tasas de reprobación y deserción, como se mencionó en la VII Cumbre de las Américas realizada el 10 y 11 de abril del 2015, a través de Varela Juan Carlos Presidente de Rectores que la región de América Latina y el Caribe se reflejan "serias debilidades en formación y capacitación para el trabajo, el desarrollo y aplicación de tecnología, hay además una Páginas 60-78 escasa inversión en innovación. La cobertura de la región universitaria representa un gran desafío debido al alto nivel de deserción que se registra en la región universitaria, y que 1 de cada 10 universitarios logra terminar los estudios completos" (González P. J., 2015). Entonces el estudio de la tutoría y su situación actual además de lo mencionado cobra importancia porque:

1. La tutoría es considerado como uno de los factores para lograr la calidad educativa (Carnicero et. Al. 2004; Castro C.R.A., 2014) y es parte del sistema educativo (Castro C.R.A. 2009, 2014 y Bordieu P., 1991) porque comprende un acompañamiento académico al estudiante para que esta pueda terminar su proceso de formación profesional. Además que la calidad educativa, permite reconocer la existencia del tutor vista como "la persona competente que tiene la capacidad de resolver problemas en el proceso de enseñanza y aprendizaje del estudiante que se encuentra en un trayecto formativo" (Tejada, 1999 en Castro C.R.A., 2014) y que el mismo contribuye en la reducción de la tasa de deserción y el incremento de la baja eficiencia de titulación y repitencia universitaria.

2. Cuando la calidad se relacionada con la educación superior ésta se considerada como un elemento determinante del desarrollo pedagógico, humano y económico de un país, ya que el hecho de lograr que la educación sea de calidad implica una relación entre el estudiante y el docente y/o profesor como outputs y los procesos como inputs, donde el aprendizaje dada a nivel de estudiante y a nivel de sistema proporciona los mecanismos de aprendizaje (UNESCO - OIE, 2008) como también aceptan (Lockheed M.E., 1991). También la calidad está relacionada con la función de producción educativa, Cano C.C.R.(2008) muestra que las variables de la función de producción para medir la calidad de la educación son el ingreso y el consumo "basado en el trabajo de Ben-Porath (1967) en Cano C.C.R. (2008) quien considera variables, como el valor de los ingresos futuros, y en los consumos, al capital humano graduado, activos, los costos universitarios, 
concluyendo que la calidad de la educación se dará si existe una participación del individuo", De esta manera la calidad de la educación está relacionada con la tutoría, por tanto, se puede considerar como instrumento fundamental para mejorar la calidad y la eficacia de la enseñanza, concibiéndose a la tutoría como un medio para desarrollar el proceso de orientación educativa.

3. Las causas de la deserción universitaria según el Informe sobre Educación Superior en América Latina y el Caribe 2000 -2005 (UNESCO, 2006) "están relacionadas con las condiciones socioeconómicas tanto del estudiante como del grupo familiar, las del propio sistema e institucional, las personales y las de orden académico previo". Es necesario superar o reducir las tasa de deserción y la repitencia y contrastar a partir de la "aplicación y de mejoras a un nivel del sistema de educación, institucional y académico (otorgar tutorial integral al estudiante)y a un nivel pedagógico"(UNESCO, 2006) (ver datos sobre deserción grafica 1). Asimismo, Cambours de Donini et.al., (2015) y la UNESCO (2006) muestran cuán importante es otorgar tutorial integral al estudiante y realizar un seguimiento estudiantil, incrementar la autoestima, el autoconocimiento para reducir las tasas de deserción, (Ver gráficos 1,2 y 3

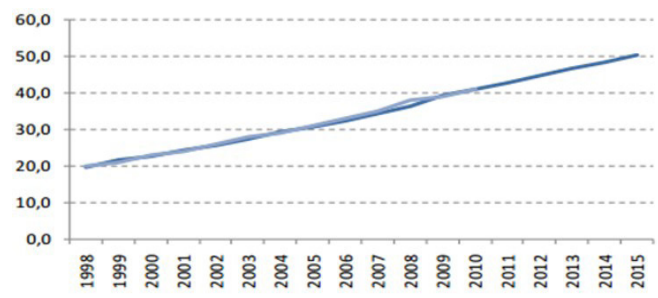

Grafico 2. Número de estudiantes en Educación Superior por cada 100.000 habitantes

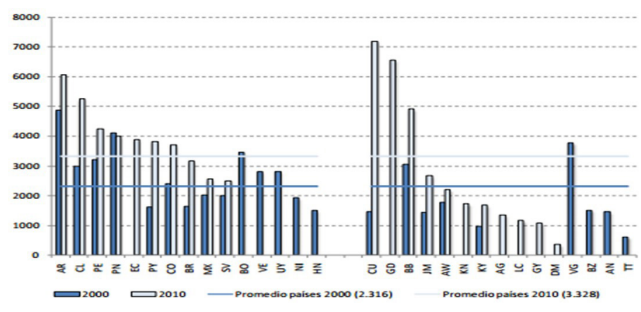

Fuente: Base de datos UNESCO - UIS, 2015 Gráfica 3. Conclusión de la educación terciaria (población de 25 -29 años)

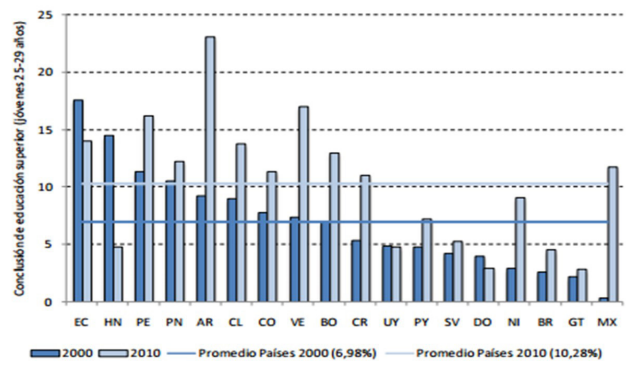

Fuente: Base de datos CEPAL, 2015

- Los Reglamentos de las Universidades, normalizan las tutorías en todo el proceso de formación en algunas y en otras en el momento de la titulación en sus diferentes modalidades de graduación propuestas por la Educación Superior Universitaria, resaltando la necesidad de apoyar los procesos educativos, no solo con la actividad didáctica tradicional, sino también acompañando los procesos de enseñanza aprendizaje investigación y hasta en lo personal, para dar solución a los problemas de aprendizaje y encontrar aplicaciones prácticas a los diferentes conocimientos que pueden ser plasmados en producciones científicas. Así, se convierte en importante el papel del docente universitario en el acompañamiento de tutorías a sus dirigidos, como menciona Díaz V.M. (2002) y Ariza et.al., (2005), asimismo, se debería considerar para su estudio los distintos puntos de vista que mencionan Hobson, et al, (2009); Wang J. y Odell (2002), así, desde "la psicología cognoscitiva de habilidades (Anderson J., 2006); Leinhardt, Young, y Merriman, 1995; Tomlinson P., 1998), desde las teorías neo-Vigostianas y socioculturales (Edwards A. y Collison J., 1996; Rogoff B., 1995; Tharp R. y Gallimore R., 1988; Wertsch J., 1991), desde la práctica reflexiva (Dewey J., 1933; Schön D, 1983, 1987; Zeichner K., 1994), desde el aprendizaje situado (Brown J., Collins A. y Duguid P., 1989; Greeno i., CollinsS. y Resnick L., 1996), desde el conocimiento de destrezas (Grim- 
mett P.P. y MacKinnon A,.M., 1992; Leinhardt et.al. , 1990), desde el conocimiento personal práctico (Doyle W., 1990; Elbaz (Fensternacher, 1994)F., 1983) y desde el razonamiento práctico (Fenstermacher G., 1986, 1994). No obstante, el problema de establecer una teorización de la tutoría en el Practicum persiste. De estas perspectivas, "se sitúa en la práctica reflexiva citado en Gid, A. (2010).

- En este contexto, el estudio sobre las tutorías, tanto a nivel conceptual como aplicado en el ámbito de la Educación Superior Universitaria permite contrastar elementos relacionales entre tutoría y calidad respondiendo a preguntas como: ¿̇porque sería importante estudiar la tutoría?, ¿̇la tutoría contribuye a la mejora o logro de la calidad educativa? ¿Qué elementos intervienen en ese proceso? ¿Puede considerarse la tutoría un mecanismo de calidad educativa? ¿De qué manera permite reducir las tasas de deserción y repitencia?

- El objetivo es determinar si la tutoría es uno de los elementos determinantes para lograr la calidad en el proceso de enseñanza y aprendizaje en el sistema universitario de la educación superior a partir del estudio teórico y práctico de la función, de los modelos, del papel o rol y de los factores medibles del tutor

- Se plantea demostrar las siguientes hipótesis de trabajo: H1. Que la definición de tutoría está asociada en la Educación Superior a las acciones y funciones del docente tutor en aula. H2. El modelo de tutoría está relacionado con los modelos académicos de la Universidad y del Sistema de Educación Superior y de las políticas educativas de cada País. H3. Los factores medibles de la tutoría son importantes para la calidad educativa en la Educación Superior Universitaria. H4. La tutoría contribuye en la reducción de la repitencia y la deserción principalmente.

\section{MATERIALES Y METODOS}

La investigación asume el método deductivo y un enfoque cualitativo - descriptivo, que contempla ámbitos, como: la realización de una revisión bibliográfica conceptual sobre las tu- torías, una revisión documental de los estudios e investigaciones realizadas sobre la temática de las tutorías en instituciones de educación superior en países de América Latina como Chile Ecuador, Argentina y México y el estudio de un caso empírico sobre la tutoría en la Universidad boliviana a fin de comparar elementos comunes y diferenciales. En el caso de Bolivia se aplica el estudio a la planta docente de la Universidad Técnica de Oruro en su carrera de Administración de Empresas, comprende un análisis cualitativo a través de la utilización del método Delfhi a 13 docentes y 30 estudiantes del décimo semestre y como instrumento de recolección de información se utilizó el cuestionario (Baker, 1997 en Ávila B. H. L., 2006).

\section{RESULTADOS \\ Situación actual de la tutoría en la Educa- ción Superior Universitaria}

Los hallazgos teóricos a partir de la revisión de estudios realizados en distintos países de América Latina, permite conocer cuál es la situación actual a partir de dar una respuesta a la $\mathrm{H} 1$. Que la definición de tutoría está asociada en la Educación Superior a las acciones y funciones del docente tutor en aula, en función a los siguientes elementos

La definición de la tutoría académica universitaria

Distintos autores como las de la tabla 1, mencionan que las tutorías han tomado y seguirán tomando importancia en el proceso de enseñanza aprendizaje en la Educación Superior Universitaria, porque contribuyen al logro de los fines y objetivos de la institución universitaria y por ende del sistema educativo, como menciona (Tinto, 1992 en Narro et.al., 2013) "que no solo permite garantizar la retención y permanencia del estudiante sino asegurar el pleno desarrollo intelectual y social" y que por lo tanto, desde esta perspectiva "resalta la importancia que tiene el sistema tutorial "(Narro et.al., 2013).

Conocer como se viene definiendo y aplicando la tutoría en la actividad académica implica revisar los distintos estudios y casos sobre este 
tema y tras realizar ese recorrido bibliográfico, se llega a identificar distintas formas de conceptualización y aplicaciones de la tutoría en la Educación Superior Universitaria, es así, que a partir de estos hallazgos se intenta comprender el sentido de la tutoría en una Universidad, sobre todo cuando ésta viene relacionada en la formación académica del estudiante y termina con el proceso de titulación como última fase de la formación pre gradual. Los aportes a este análisis vienen dadas por los autores que aparecen en la tabla 1 .

Tabla 1. Aporte teórico sobre las definiciones de las tutorías en la Educación Superior Universitaria

\begin{tabular}{lll}
\hline$N^{\circ}$ & \multicolumn{1}{c}{ Autores } & \multicolumn{1}{c}{ Definición } \\
\hline 1 & Levinson et. Al., & $\begin{array}{l}\text { Proceso de desarrollo importante en la madurez con beneficios para el tutor } \\
\text { y tutorando } \\
\text { Señala que el concepto de tutoría es más amplio que el proceso de E-A } \\
\text { centrado en la docencia, pero no separable de este. } \\
\text { Destaca que la acción tutorial tiene que devenir como la concreción de un } \\
\text { proceso orientador integral y propio. } \\
\text { La actividad del profesor tutor encaminada a propiciar un proceso madurativo } \\
\text { permanente, a través del cual el estudiante universitario logre obtener } \\
\text { y procesar información correcta sobre sí mismo y su entorno, dentro de } \\
\text { planteamientos intencionales de toma de decisiones razonadas: integrar la } \\
\text { constelación de factores que configuran su trayectoria vital; afianzar su auto } \\
\text { concepto a través de experiencias vitales en general y laborales en particular; } \\
\text { desplegar las habilidades y actitudes precisas, para lograr integrar el trabajo } \\
\text { dentro de un proyecto de vida global. }\end{array}$
\end{tabular}

5 González, 2001

Acción docente de orientación con componentes pedagógicos y psicológicos, realizada por profesores - tutores con la finalidad de participar en la formación integral del estudiante potenciando su desarrollo como persona y como estudiante y su proyección social y profesional. De este modo, se considera el tutor como un elemento personal y funcional del proceso educativo con funciones de defensa, ayuda, preocupación, resolución de problemas de la clase, de mediación o representación, etc.

6 Wenger, 2001

Proceso formativo de carácter socio cognitivo, personalizado y dirigido a convertir a los novatos en individuos competentes, mediante su integración a comunidades de práctica y redes de experto, que resuelven en ambiente dinámicos y complejos, crean y recrean la acción profesional y, en su caso, generan conocimiento avanzado.

7 Álvarez, 2002

"Un recurso, una estrategia para que los estudiantes afronten de forma satisfactoria su proceso formativos y afronten, de manera adecuada, la toma de decisiones, los procesos de transición académica y la vida socio laboral activa de manera autónoma y responsable.

$8 \quad$ Almajano, 2002

La tutoría persigue que el profesor tutor escuche a sus alumnos y a partir de lo que oye pueda ayudarles a ejercer su libertad, sugiriéndole caminos y valorando para cada uno de ellos las ventajas y las dificultades.

$9 \quad$ Planas, 2002

La función tutorial ha de identificarse con la función docente, con el sentido de formar parte de ésta dentro de un planteamiento educativo integral y altamente personalizado.

10 Soler, 2003

Proceso con el cual una persona con más experiencia (el mentor) enseña, aconseja, guía y ayuda a otra (el tutelado) en su desarrollo personal y profesional, invirtiendo tiempo, energía y conocimientos. 
Coriat \& Sanz, 2005

Gid, 2010
Es una actividad de carácter formativo que incide en el desarrollo integral de los estudiantes universitarios en su dimensión intelectual, académica, profesional y personal.

Los profesores son formadores y ejercen esa tutoría (una especie de acompañamiento y guía del proceso de formación) del alumnado. La tutoría adquiere así un contenido similar al de función orientadora o función formativa de la actuación de los profesores.

La tutoría universitaria se entiende como una acción de intervención formativa destinada al seguimiento académico de los estudiantes, desarrollada por profesores como una actividad docente más, si bien con el apoyo, coordinación y recursos técnicos facilitados por el profesorado especializado o personal técnico.

Mediante la tutoría el profesor tutor facilita al estudiante una ayuda, basada en una relación personalizada, para conseguir sus objetivos académicos, profesionales y personales a través del uso de la totalidad de recursos institucionales y comunitarios.

La tutoría es una actividad formativa realizada por el profesor tutor encaminada García et al, 2005 al desarrollo integral (intelectual, profesional y humano) de los estudiantes universitarios. Se pretende que los estudiantes vayan adquiriendo no sólo saberes sino además competencias que le permitan auto dirigir su proceso de aprendizaje a lo largo de la carrera y durante su ejercicio profesional.

La tutoría supone tutela, guía, asistencia, apoyo y ayuda a una persona mediante la orientación y el asesoramiento.

Que la tutoría aporta beneficios tanto para los tutores como para los tutorandos. Como se ha puesto de manifiesto, los principales beneficios para los tutores se refieren al desarrollo profesional y personal, al aprendizaje (reflexión) y la satisfacción por su trabajo. Por lo que se refiere a los tutorandos, los beneficios hacen referencia, principalmente, al «apoyo» para su desarrollo profesional, la ayuda para realizar aprendizajes (reflexión)y la socialización. No obstante, pese a los beneficios que la tutoría

Narro, 2013 La tutoría implica procesos de comunicación y de interacción de parte de los profesores; implica una atención personalizada a los estudiantes, en función del conocimiento de sus problemas, de sus necesidades y de sus intereses específicos. Es una intervención docente en el proceso educativo de carácter intencionado, que consiste en el acompañamiento cercano al estudiante, sistemático y permanente, para apoyarlo y facilitarle el proceso de construcción de aprendizajes de diverso tipo: cognitivos, afectivos, socioculturales y existenciales.
Las distintas conceptualizaciones muestran que las tutorías tienen diferentes acepciones, como la que está relacionada entre la institución, el docente y el estudiante. Esto significa que la tutoría es considerada como un conjunto de acciones orientadoras y paralelas a la práctica instructiva. En cambio otros entienden como una acción que forma parte de las actuaciones del propio docente y que por tanto es inherente a la función educativa (Álvarez P.P., 2008). En este sentido, se puede apreciar que existen dos formas de referirse a la tutoría. La primera, hace hincapié al docente cuando se hace referencia a la tutoría universitaria (Levinson, et.al., 1978) y la otra, al profesor tutor cuando se hace referencia a las labores de asesoramiento (Narro, et.al., 2013). Claro que cada una de estas visiones, responden a los modelos de tutorías; sin embargo, es importante considerar que si bien algunas definiciones resaltan las labores académicas y formativas, también se puede observar el de desarrollo personal, social y profesional. Al margen de ello, también es de relevancia mencionar que algunas definiciones 
se enmarcan dentro de las competencias de los futuros profesionales, ya que se considera que lo importante es desarrollar competencias que sean de utilidad para el futuro profesional al margen de ayudar, enseñar o acumular saberes, lo confirman Alañón R., 2000; Rodríguez E., 2004; Sancho G.J., 2000; Almajano P. 2000; Coriat y Sanz, 2005).

\section{El rol del tutor en la educación superior universitaria}

Distintos autores mencionan que el tutor es quien representa a alguien, quien cuida, protege, vela o tiene bajo su responsabilidad a otro. Es decir "quien guía a alguien" y se define al tutor como el profesor que tutela la formación humana y científica de un estudiante y le acompaña en sus procesos de aprendizaje" (Lázaro A., 2003 en García N., 2008 y Lázaro, A y Asensi J., 1986), asimismo, el tutor es un profesor encargado de un grupo de alumnos en algo más que en dar clases; en ayudar a la decisión del grupo y de cada alumno para que se realicen como personas; en ayudar al desarrollo del grupo y de cada alumno individual y socialmente; en apoyar al conocimiento; en la adaptación y dirección de sí mismo para lograr el desarrollo equilibrado de sus personalidades y para que lleguen a participar con sus características peculiares de una manera eficaz en su formación profesional. Es importante resaltar la diferencia entre el tutor y el asesor; el primero es quien acompaña, guía y asiste al estudiante, orientado en el desarrollo de la investigación para el aprendizaje, en cambio el asesor es el especialista en una rama de la ciencia, donde el alumno puede consultar sobre temas disciplinares de su competencia o interés. Lo que significa que las universidades vienen considerando tutores a profesores que tienen una asignatura, y como parte de su responsabilidad es desarrollar procesos colectivos de organización de aprendizajes utilizando entornos presenciales o virtuales con el fin de cumplir esa función tutorial y de asesoramiento, gestionando el aprendizaje práctico con tutoría profesional y académica e insertando al estudiante en contextos reales de la profesión.

\section{La función tutorial en la educación su- perior universitaria}

Distintos autores están de acuerdo que la función tutorial consiste en "ofrecer una orientación académica pro-activa que implique activamente al estudiante en el fomento de su aprendizaje" (Boud et.al.. 1996); esta función debe ser centrada en una función tradicional, que consiste en primer lugar en un apoyo puntual y tiene el objetivo de proporcionar información académica, profesional y aclarar ideas, acompañar en el desarrollo académico mediante la asesoría, utilizando técnicas de enseñanza apropiadas, presenciales o virtuales, como metodología se utiliza la tutoría individual o grupal, modalidad presencial In situ o virtual sincrónico o asincrónico, lo que permite resolver dudas, puede desarrollarse dentro y fuera del aula de clases, la estrategia que se utiliza es la resolución de preguntas, aclaración de dudas, información puntual, además de que la tutoría puede ser esporádica donde intervienen tanto el Profesor y el estudiante, y la participación puede ser voluntaria; en segundo lugar se integra la acción docente y la formación del estudiante, tiene como objetivo orientar y guiar en las tareas del proceso de aprendizaje, el rol que cumple el tutor es el de construir conocimiento, utiliza una metodología personalizada o grupal tanto dentro o fuera del aula, voluntaria y/o obligatoria, puede ser presencial o virtual, como estrategia utiliza la detección de necesidades, adquisición de habilidades de estudio, información académica, rendición de cuentas, orientación sobre estudios específicos, la tutoría es continuo y los agentes que intervienen son el profesor y el estudiante.

Lo que significa que los tutores desarrollan competencias culturales, pedagógicas, investigadoras, tecnológicas e interpersonales como también lo enfatiza García N.(2008) y que el tutor se convierte en un agente de desarrollo 
académico que permite lograr los objetivos educativos y contribuir en el logro de la calidad tanto como insumo o producto de la función de producción, demostrando de esta manera que la tutoría implica acciones y funciones del docente tutor en aula para lograr un rendimiento adecuado en el proceso de enseñanza y aprendizaje, por tanto como efecto se traduce en la reducción de la deserción y repitencia.

\section{Los modelos académicos y los modelos de tutoría en la Educación Superior Universi- taria}

Los resultados de la investigación muestran que H2. El modelo de tutoría está relacionado con los modelos académicos de la Universidad y del Sistema de Educación Superior y de las políticas educativas de cada País.

Los modelo de tutoría en la educación superior universitaria.
La tutoría llega a la modelización desde que es insertado en la currícula académica de las instituciones educativas universitarias, esta modelización se relaciona con el modelo académico de cada Institución de Educación Superior, puede variar en función de los objetivos institucionales, de la visión y misión de la carrera y del perfil profesional, del contexto social y económico de un país, se relacionan con el tipo de modelo pedagógico, por ello la tutoría tiene una alta relación con el logro de la calidad educativa, ya que los objetivos de la educación superior se refleja en los códigos y leyes de educación de cada país. Así, desde esta perspectiva se puede identificar que existen alrededor de siete modelos de tutorías aplicados en la Educación Superior Universitaria (ver figura 1).

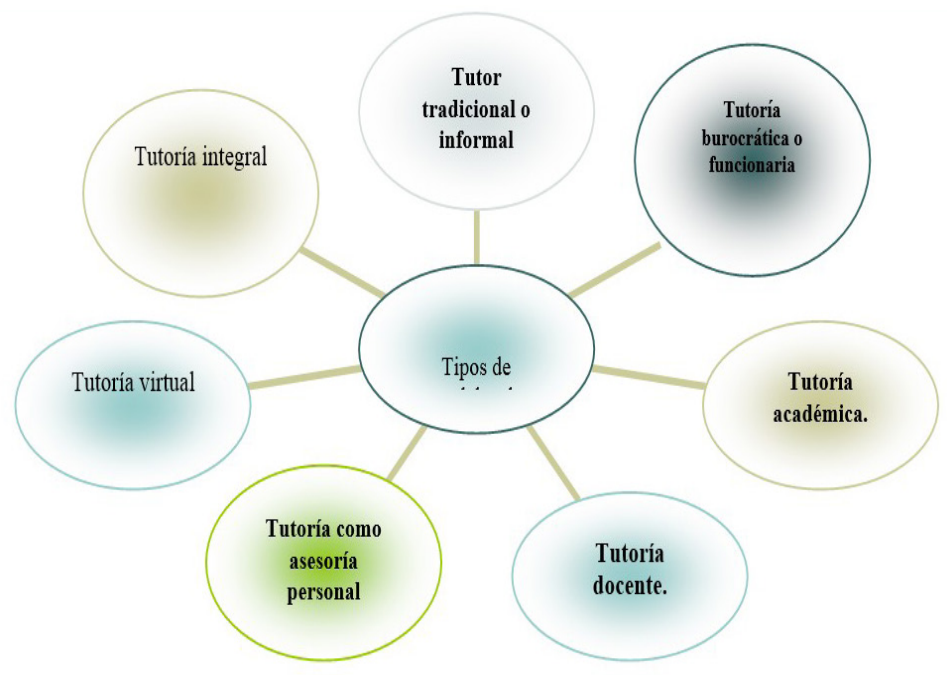

Figura 1. Modelos de tutorías en la Educación Superior Universitaria 
Cada uno de estos modelos consiste en lo siguiente (ver tabla 2): Tabla 2. Conceptualización de los modelos académicos de tutorías

\begin{tabular}{|c|c|}
\hline $\begin{array}{l}\text { Tutoría } \\
\text { académica }\end{array}$ & $\begin{array}{l}\text { Es aquella que se centra en el ámbito académico, circunscribiéndose al ámbito científico. El tutor universitario } \\
\text { asesora respecto a estudios y asignaturas, bibliografías y fuentes de documentación, elaboración de } \\
\text { trabajos de curso, realización de investigaciones o trabajos de campo, etc. }\end{array}$ \\
\hline $\begin{array}{l}\text { Tutoría docente, } \\
\text { de tradición } \\
\text { anglosajona }\end{array}$ & $\begin{array}{l}\text { Pretende ser un tipo o forma de docencia especial que complete y amplíe la ofrecida en las clases } \\
\text { convencionales: docencia en pequeños grupos, realización de seminarios, profundización en algún tema } \\
\text { monográfico, realización de debates sobre cuestiones problemáticas, trato/relación especial (participación, } \\
\text { estilo). }\end{array}$ \\
\hline $\begin{array}{l}\text { Tutoría como } \\
\text { asesoría } \\
\text { personal }\end{array}$ & $\begin{array}{l}\text { Procura ofrecer una atención personalizada al alumno, globalmente considerado, en todos sus aspectos } \\
\text { y necesidades intelectuales, sociales, académicas y personales, transmisión de información, orientación, } \\
\text { asesoramiento académico, personal, social y vocacional, atención a cualquier problema o ámbito intelectual, } \\
\text { afectivo, social, familiar y profesional. Supone la aceptación mutua profesor/alumno, transmisión de } \\
\text { valores, actitudes y estilo de la institución como filosofía de vida, convicciones, etc. }\end{array}$ \\
\hline Las tutorías & $\begin{array}{l}\text { Este tipo de tutoría generalmente se viene aplicando en universidades que cuentan con programas virtuales, } \\
\text { donde la tutoría virtual se convierte en un elemento fundamental para lograr los objetivos académicos. }\end{array}$ \\
\hline $\begin{array}{l}\text { La tutoría } \\
\text { integral }\end{array}$ & $\begin{array}{l}\text { Consiste en convertir al estudiante en personas competentes a través de la integración a la comunidad, } \\
\text { donde el tutor facilita la integración en redes de expertos, la creación de una acción de tutoría profesional } \\
\text { hasta que el tutorado logre generar un conocimiento avanzado sobre la realidad de su comunidad. (De la } \\
\text { Cruz, G, García, T. y Abreau, L. 2006, p. } 1367-1368\end{array}$ \\
\hline
\end{tabular}

Ahora bien, la práctica de la tutoría presenta dos tipos de tutorías (Tabla 3), la primera relacionada con la tutoría académica y de aula, que consiste en apoyar al estudiante durante el proceso de su formación profesional; y la segunda con la titulación que consiste en apoyar al estudiante durante la elaboración de su trabajo de investigación que generalmente viene vinculada con las modalidades de titulación y responden a los modelos académicos de las universidades.

Tabla 3. Clasificación de las tutorías

\begin{tabular}{|c|c|c|}
\hline $\mathrm{N}^{\circ}$ & Tipo de tutorías & Características \\
\hline 1 & \multicolumn{2}{|c|}{ Tutorías vinculada a una materia } \\
\hline 1 & $\begin{array}{l}\text { Las tutorías } \\
\text { Complementarias o } \\
\text { reactivas: }\end{array}$ & $\begin{array}{l}\text { Consiste en que la tutoría es convencional. Se entiende como ayuda a la enseñanza } \\
\text { presencial en la que el alumno soluciona dudas sobre el tema y el desarrollo de } \\
\text { una asignatura especifica. Son voluntarias y no suelen ser consideradas en los } \\
\text { procesos de evaluación }\end{array}$ \\
\hline 2 & $\begin{array}{l}\text { Las tutorías } \\
\text { Complementarias } \\
\text { programadas }\end{array}$ & $\begin{array}{l}\text { Estas tutorías son obligatorias y están directamente relacionadas con la evaluación } \\
\text { continua de una materia. }\end{array}$ \\
\hline 3 & $\begin{array}{l}\text { Las tutorías } \\
\text { Fundamentales }\end{array}$ & $\begin{array}{l}\text { Estas están previstas para el seguimiento de las prácticas en empresas que } \\
\text { complementarán la formación del estudiante, así como para ayudarles en la } \\
\text { realización de la memoria fin de prácticas o en el trabajo de fin de grado. }\end{array}$ \\
\hline II & Tutorías relacionadas con la & titulación \\
\hline 1 & $\begin{array}{l}\text { Las tutorías personales y } \\
\text { profesionales }\end{array}$ & $\begin{array}{l}\text { Se trata de un modelo implantado en los países anglosajones, en el que se } \\
\text { mezclan recomendaciones académicas, profesionales y personales. }\end{array}$ \\
\hline 2 & Las tutorías académicas & $\begin{array}{l}\text { Suponen un servicio de orientación en aspectos académicos y profesionales, en } \\
\text { el que los tutores llevan a cabo actividades individuales y grupales de carácter } \\
\text { académico-transversal. }\end{array}$ \\
\hline
\end{tabular}


Lo que significa que la función del docente es tutorial y se encuentra vinculada a reforzar, acompañar, facilitar, orientar y guiar a que los alumnos construyan su proyecto de vida, potenciando el trabajo, autónomo, critico, reflexivo y propositivo, con capacidad para innovar y emprender.

Así, los modelos académicos y educativos son los que determinan el modelo de tutoría académica en una Unidad Académica (Facultad, Carrera o Centro) en la Educación Superior Universitaria entendiéndose por modelo académico "una concepción dinámica que articula congruentemente el horizonte de la visión y orienta las acciones a seguir en el proceso educativo, asegurando el cumplimiento de la misión y en un anhelo de mejora de vida, también se conceptualiza al modelo educativo como esquema teóricos de un sistema o una realidad que se elabora para facilitar su comprensión y estudio. traduce en organización académica y diseño curricular, el compromiso de la institución con su modelo educativo" (Bolaños, E. 2008 , p. 25); y por modelo educativo "la concreción en términos pedagógicos, de los paradigmas educativos que una institución profesa y que sirve de referencia para todas las funciones que cumplo (docencia, investigación, extensión, vinculación y servicios), a fin de hacer realidad su proyectos educativo, esta debe estar sustentado en la historia, valores profesados, la visión, la misión, la filosofía, objetivos y finalidades de la institución" (Tunnerman, 2009) en Bolaños, E. (2008, p. 15). Asi, el modelo académico permite la construcción del modelo educativo, por lo que los modelos educativos son visiones de teorías o enfoques pedagógicos que orientan en la elaboración y análisis de los programas de estudios, en la sistematización del proceso de enseñanza y aprendizaje.

En este contexto, la tutoría en los modelos académicos puede variar en función a los objetivos institucionales de la Universidad como de Estado, y el modelo educativo representa las relaciones previstas dentro del proceso educativo (enseñanza-aprendizaje), y permite comprender las limitaciones y potencialidades del sistema educativo.

En este marco, las Universidades Latinoamericanas tienen como base un modelo académico,

Páginas 60-78 que está relacionado con la enseñanza (Procesos) aprendizaje (Contenidos) entre el educador (entregar, enseñar a enseñar) y el educando (toma, aprende a aprender), la cual debe ser evaluada. De esta forma, los modelos académicos que se aplican son: el modelo tradicional que consiste en la elaboración de un programa de estudios y es de tipo lineal; el modelo por competencias, que consiste en elaborar el programa en función a las necesidades del mercado y es lineal y; el modelo de tipo matricial, que es de tipo lineal vertical, que considera la elaboración de un programa en función a las necesidades pero se comparte las asignatura entre dos o más especialidades, donde el modelo tiene una aplicación universal y la tutoría es un proceso de acompañamiento y disciplinar académico durante toda la trayectoria de formación; la acción de apoyo tutorial es un acompañamiento especifico disciplinar, metodológico, psicológico, individual en puntos determinados en la trayectoria de la formación, como en el momento de la titulación que viene caracterizada por el apoyo en la elaboración de un trabajo de investigación principalmente.

Así, estos modelos de tutoría contribuyen a los modelos educativos, ya que, si una unidad académica no cuenta con el modelo educativo ni académico, tampoco contará con el modelo de tutoría, por tanto, es fundamental que el aprendizaje, investigación y la tutoría académica se interrelacionen para lograr la calidad educativa a través de la formación de profesionales competentes y de esta manera contribuir al desarrollo humano y económico de un país.

\section{Factores medibles de la tutoría y su relación con la Calidad educativa}

En este acápite se presenta los resultados obtenidos de los estudios de caso realizados sobre las tutorías en países de América Latina, y reflexionar sobre los alcances y límites que tiene la tutoría, a fin de explicar que la tutoría es un elemento determinante para lograr la calidad de la educación universitaria. En este contexto se presenta los casos estudiados y su análisis comparativo sobre la tutoría a fin de contrastar la H3. Los factores medibles de la tutoría son importantes para la calidad educativa en la Educación Superior Universitaria.

Los resultados de la investigación empírica aplicada en la Universidad Técnica de Oruro en su 
carrera de Administración de Empresa, muestran que los elementos que caracterizan a la tutoría está dada por la conceptualización de la tutoría, el tipo de tutoría que se aplica, los modelos de tutorías, el rol, papel y función del tutor, modalidades de titulación principalmente, como se observa en la tabla 4.

Tabla 4. Cuadro comparativo de resultados de los cuestionarios

\begin{tabular}{|c|c|c|}
\hline Elementos & Resultados Docentes & Resultados Alumnos \\
\hline $\begin{array}{l}\text { Conceptualización de } \\
\text { las tutorías }\end{array}$ & $\begin{array}{l}\text { Se conceptualiza la } \\
\text { tutoría como "un } \\
\text { asesoramiento, una } \\
\text { orientación adecuada } \\
\text { para la elaboración } \\
\text { de un trabajo de } \\
\text { investigación en sus } \\
\text { diferentes modalidades". }\end{array}$ & $\begin{array}{l}\text { Por tutoría se entiende fundamentalmente como: - } \\
\text { Corrección de dudas que tiene el alumno, - Guía } \\
\text { sobre cómo hacer los trabajos de investigación. - } \\
\text { Persona responsable como guía para realizar un } \\
\text { trabajo de investigación. Esto significa, que los } \\
\text { alumnos observan a un tutor como un guía en la } \\
\text { realización de un trabajo de investigación, que } \\
\text { está relacionado con el trabajo de fin de estudios. } \\
\text { Y no se relaciona con otras materias que no estén } \\
\text { relacionadas con el trabajo de fin de curso. }\end{array}$ \\
\hline Tipo de tutoría & $\begin{array}{l}\text { La tutoría parte de un } \\
\text { criterio personalizado del } \\
\text { tutor a uno grupal. }\end{array}$ & $\begin{array}{l}\text { Se califica que la actividad de tutoría desarrollada } \\
\text { por el docente en general como regular. Y } \\
\text { generalmente es grupal. }\end{array}$ \\
\hline Modelos de tutoría & $\begin{array}{l}\text { Existe un } \\
\text { desconocimiento de los } \\
\text { modelos de tutoría. }\end{array}$ & \\
\hline $\begin{array}{l}\text { El rol o papel de un } \\
\text { tutor. }\end{array}$ & $\begin{array}{l}\text { El rol del tutor se } \\
\text { encuentra enmarcado en } \\
\text { la orientación dirigida al } \\
\text { alumno, que depende } \\
\text { de la modalidad de } \\
\text { titulación del alumno. }\end{array}$ & $\begin{array}{l}\text { Los alumnos en general consideran que el rol del } \\
\text { tutor es: - Orientar al alumno. - Guiar al alumno, } \\
\text { ayudar a resolver los cuestionables que se tiene } \\
\text { en la elaboración del trabajo de investigación. - } \\
\text { Relación tutor - alumno. Lo que significa que el } \\
\text { alumno espera que el tutor se convierta en su guía } \\
\text { para culminar la carrera. }\end{array}$ \\
\hline Función del tutor. & $\begin{array}{l}\text { El tutor tiene la función } \\
\text { de apoyar, guiar, orientar, } \\
\text { facilitar, en el proceso de } \\
\text { enseñanza aprendizaje }\end{array}$ & $\begin{array}{l}\text { La función del tutor está relacionada con el papel } \\
\text { que realiza en la tutoría y que esta debe satisfacer } \\
\text { las necesidades del estudiante. }\end{array}$ \\
\hline $\begin{array}{l}\text { Acuerdo con las } \\
\text { modalidades de } \\
\text { titulación. }\end{array}$ & $\begin{array}{l}\text { Existente una aceptación } \\
\text { total. }\end{array}$ & $\begin{array}{l}\text { La mayoría de los alumnos están de acuerdo con } \\
\text { las modalidades de titulación existente. }\end{array}$ \\
\hline $\begin{array}{l}\text { Cambios que } \\
\text { debería de realizarse } \\
\text { en las modalidades } \\
\text { de titulación. }\end{array}$ & $\begin{array}{l}\text { No deberían realizarse } \\
\text { ningún cambio a las } \\
\text { diferentes modalidades } \\
\text { existentes. }\end{array}$ & $\begin{array}{l}\text { La mayoría considera que no deben realizarse } \\
\text { ningún cambio. }\end{array}$ \\
\hline
\end{tabular}


de modalidades de tutorías, como son la tutoría personalizada, grupal, ocasional y obligatoria cuyas características son: 1) Tutoría personalizada, consiste en una interacción directa entre el docente tutor y el estudiante, siendo el estudiante el que debe acudir a las entrevistas con su tutor tantas veces considere necesarias en los horarios programados y publicados como parte de la currĺcula del estudiante; 2) Tutoría grupal, que consiste en una actividad académica en la que interactúan de forma directa un pequeño grupo de estudiantes con un docente tutor, donde el estudiante debe acudir a las entrevistas con su tutor tantas veces sean necesarias en los horarios programados como parte de su currícula y; 3) Tutoría ocasional, no se encuentra regulada por el sistema de educación superior pero que se viene practicando en todo el sistema universitario y que no es obligatoria como las anteriores, consiste en una interacción entre el docente de una asignatura y el estudiante, a fin de disolver dudas y que el estudiante pueda retroalimentar sus trayectorias de aprendizaje. Estos elementos permiten conocer cuál es la situación actual en la que se encuentra la tutoría, como se vienen desarrollando, se alcanzan los objetivos, existe satisfacción por parte del tutorado, ha disminuido la repitencia y deserción escolar, que cambios debería realizarse, 4) tutoría obligatoria, que viene dirigida a los alumnos de los dos últimos semestres para el logro de su titulación, con características similares a la tutoría personalizada y grupal.

Factores determinantes para la medición de la tutoría

La teoría menciona que existen distintos tipos de tutorías que el docente puede desarrollar, conocer ello, implica evaluar la tutoría a partir de algunos indicadores. Las variables consideradas para tal efecto son las que se presenta en la tabla 5 y los resultados obtenidos en cada una de ellas. Para ello, se aplica la escala de Likert con una valoración del 1 al 3. Los resultados de la investigación, muestran que las tutorías que se vienen aplicando se encuentran enmarcadas en una tutoría grupal en un $100 \%$ en una tutoría personalizada en un 50\% como se presenta en la Tabla 5.

Tabla 5. Variables que permiten medir la tutoría por parte de los docentes (promedio) Los resultados muestran que existen cuatro tipos

\begin{tabular}{|c|c|c|c|c|c|}
\hline Aspectos & $\begin{array}{c}\text { Tutoría } \\
\text { personalizada }\end{array}$ & $\begin{array}{l}\text { Tutoría } \\
\text { grupal }\end{array}$ & $\begin{array}{c}\text { Tutoría } \\
\text { entre } \\
\text { iguales }\end{array}$ & $\begin{array}{l}\text { Auto } \\
\text { orienta } \\
\text { ción }\end{array}$ & $\begin{array}{l}\text { Tutoría } \\
\text { virtual }\end{array}$ \\
\hline \multicolumn{6}{|l|}{ FACTORES DE FUNCION TUTORIAL } \\
\hline Trato profesor estudiante & 2 & 3 & 2 & 1 & \\
\hline Tiempo de actividad & 2 & 3 & 2 & 1 & \\
\hline Oportunidades de responder & 2 & 3 & & 1 & \\
\hline Oportunidades de corrección de errores & 2 & 3 & 2 & 1 & \\
\hline Oportunidades de ayudar y animar & & 3 & & 1 & \\
\hline Oportunidades de cooperar & 2 & 3 & & 1 & \\
\hline Motivación & 2 & 3 & 2 & 1 & \\
\hline Coste tiempo & & 3 & & 1 & \\
\hline \multicolumn{6}{|l|}{ FACTORES DE FORMACION } \\
\hline Coste en formación & & 3 & & 1 & \\
\hline Requisitos de formación previa & & 3 & & 1 & \\
\hline Requisitos de control de calidad & & 3 & & 1 & \\
\hline Preparación de material & & 3 & & 1 & \\
\hline $\begin{array}{l}\text { Choque con la práctica docente } \\
\text { tradicional }\end{array}$ & & 3 & & 1 & \\
\hline Total & $50 \%$ & $100 \%$ & $33 \%$ & $100 \%$ & $0 \%$ \\
\hline
\end{tabular}


Lo que significa, que la tutoría puede ser evaluada a partir de factores que se relaciona con; 1) La acción y función tutorial, como son: el trato del docente hacia el alumno, la predisposición de brindar un tiempo para apoyar, responder a las preguntas del estudiante, corregir errores, apoyo académico, ayudar y animar, cooperar, motivar y disponer de tiempo, y, 2) con la formación del docente que predispone la función tutorial, como son: Coste en formación, requisitos de formación previa, requisitos de control de calidad, preparación de material y el choque con la práctica docente tradicional. Estos factores de tipo académico, muestran la probabilidad de incidencia en la reducción de la deserción y repitencia, motivación en el aprendizaje y en la construcción de conocimiento nuevo.

\section{Análisis comparativo de casos para identifi- car factores diferenciales de la tutoría}

Tabla 6. Análisis comparativo de la tutoría en países de América Latina
Aporte de los estudios de caso de las Universidades de la República del Ecuador, República de Argentina y de los Estados Unidos Mexicanos Las investigaciones empíricas realizadas sobre la tutoría en las universidades del sistema educativo de la República del Ecuador, República Argentina y de los Estados Unidos Mexicanos por Molina B.I.A (2012), Gid, A. (2010); Universidades Publicas Ecuatorianas (2015), Bianculli y Marchall (2013), Reglamento de tutorías de las Universidades Ecuatorianas (2015), (Cuesta, 2014), Bianculli, Karina y Marchal, Monica (2013), (Capelari, 2009) (Aguilar, 2013) entre otros, permite comprobar la hipótesis que la tutoría desde el punto de vista teórico y práctico puede contribuir a mejorar la calidad de la educación con base en la reducción de la deserción y repitencia (ver Tabla 6).

\begin{tabular}{|c|c|c|c|c|}
\hline $\begin{array}{c}\text { FACTOR } \\
\text { COMPARATIVO }\end{array}$ & BOLIVIA & ARGENTINA & ECUADOR & MEXICO \\
\hline Tipo De Tutoría & $\begin{array}{l}\text { Tutoría personalizada } \\
\text { Tutoría grupal Tutoría } \\
\text { ocasional } \\
\text { Tutoría obligatoria } \\
\text { Tutoría de tesis }\end{array}$ & $\begin{array}{l}\text { Tutoría académica } \\
\text { Tutoría personal Tutoría } \\
\text { profesional } \\
\text { Tutoría de tesis }\end{array}$ & $\begin{array}{l}\text { Tutoría personalizada } \\
\text { compensatoria } \\
\text { Tutoría individual } \\
\text { Tutoría grupal Tutoría obligatoria }\end{array}$ & $\begin{array}{l}\text { Tutoría individual } \\
\text { Tutoría grupal }\end{array}$ \\
\hline
\end{tabular}

Conceptualización de las tutorías
Tutoría

o dispositivo pedagógico

que permite seguir,

facilitar, acompañar,

guiar, procesos

educativos.
La tutoría académica es un método de enseñanza por medio del cual un estudiante de forma individual $\circ$ un grupo pequeño de estudiantes reciben formación académica personalizada, compensatoria o complementaria de parte de los docentes a los y las estudiantes que tengan dificultades para aprender mediante los métodos convencionales $\circ$ que tienen necesidades especiales de

aprendizaje en las respectivas áreas académicas. Aptitud,

Actitud, Función

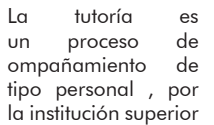

la institución superior

\begin{tabular}{llll} 
& El rol del tutor se & & \\
encuentra enmarcado en & & Asesoramiento \\
El rol o papel de un & $\begin{array}{l}\text { la orientación dirigida al } \\
\text { alumno, que depende } \\
\text { tutor }\end{array}$ & $\begin{array}{l}\text { compañamiento } \\
\text { de la modalidad de } \\
\text { titulación del alumno. }\end{array}$ & $\begin{array}{l}\text { Supervisión } \\
\text { Guía } \\
\end{array}$ \\
\hline Función del tutor & Apoyo & Apoyo & Facilitador \\
\hline
\end{tabular}

A tutoría en todo el proceso 
El resultado del análisis comparativo, permite identificar similitudes en el factor: a) tipo de tutoría, la mayoría de las unidades académicas de los países estudiados utilizan la tutoría personalizada y grupal, si existen diferencias, estas radican en la aplicación de la tutoría en el proceso educativo y en la titulación; b) en la conceptualización, existe elementos homogéneos relacionados con la función del tutor, constituyéndose el tutor en guía, asesor y acompañante; c) en el rol del tutor, existen distintas formas de entender tal es el caso de pensar que es similar a las funciones; d) en la función del tutor, se considera como apoyo, guía, acompañante, asesor; facilitador; $y$, e) en el desarrollo de la tutoría, siendo que en todas las universidades la tutoría es ejecutada en el proceso educativo para validar los logros de aprendizaje que va alcanzado el estudiante, y la forma de desarrollar es grupal e individual, en modalidad presencial y virtual, en la fase de titulación se valida las capacidades cognitivas que se declaran en el perfil de egreso.

\section{DISCUSIÓN}

Aunque Narro R. (2013) menciona "que la tutoría no es una fórmula que nos permita solucionar todos los problemas educativos, ni de tipo organizativo (escuela), ni de enseñanza o de acción profesoral, como tampoco del propio estudiante; sin embargo, es de subrayar que su adecuado desarrollo profundiza grandes beneficios y constituye una alternativa importante frente a la problemática actual de la docencia, en particular, al marcado desinterés que frecuentemente muestran los estudiantes en sus estudios y a la falta de expectativas de futuro con relación a sus carreras", el estudio llega a determinar que la tutoría académica puede constituirse en un elemento importante en el desarrollo de las actividades académicas del docente, estas las puede desarrollar dentro del aula, como en ambientes de aprendizaje diseñados en los ámbitos laborales y académicos, a fin de contribuir a la función de producción educativa y lograr de esta manera la eficiencia y eficacia de los procesos de enseñanza aprendizaje, y fortalecer las capacidades para la organización y la integración del conocimiento teórico y profesional. Por tanto, la tutoría es un factor o mecanismo que permite mejorar el proceso de enseñanza y aprendizaje y ello contribuye en la reducción de las tasas de deserción y repitencia del estudiante.

Asimismo, el análisis comparativo muestra que las universidades están conscientes de la importancia de la tutoría en el proceso de enseñanza aprendizaje, ahora bien, como está la vienen aplicando es un tema crucial, sin embargo desde el punto de vista teórico y práctico es una cuestión de aplicación del modelo educativo, y es ahí donde aparece la calidad, siendo un reto para cualquier institución educativa. Entonces desde este punto de vista la calidad está relacionada con elementos como la del prestigio, la excelencia, la función de recursos, los resultados, el cambio o valor añadido, la adecuación de propósitos, el mérito, la meta, la satisfacción. Sin embargo, la tutoría se constituye en un elemento fundamental y trascendental para lograr la calidad educativa, que interviene en procesos que pueden ser mejorados continuamente y que están asociadas al funcionamiento de la institución y al trabajo en equipo entre el docente, estudiante e institución. En definitiva, la institución educativa universitaria debe considerar elementos de cooperación tanto del estudiante, administrativos, y el docente que se constituye en un ente importante para el desarrollo del pensamiento científico de los estudiantes para la transformación de la realidad, concordante con los cambios del país y del mundo y de esta manera alcanzar las metas y objetivos institucionales.

Por lo que se puede destacar que la calidad de la educación depende también de la intervención de un tutor en el proceso de transferencia y generación de conocimiento, ya que el alumno al recibir apoyo por parte del docente, puede generar, innovar y emprender su propio proyecto de vida, y contribuir a dar solución a las necesidades de la sociedad, y es ahí donde las universidades deben implementar nuevas formas de tutorías, cambiando de un modelo individual voluntario a uno obligado, ya que la práctica de la tutoría implica un compromiso y responsabilidad por parte del tutor, turado y la institución, lo que significa que los docente deben cumplir esa función tutorial en todo el proceso de aprendizaje a fin de mejorar las capacidades profesionales, personales, sociales 
y administrativas del estudiante, porque este hecho contribuye a la reducción de la tasas de repitencia y deserción y contribuir al mejoramiento de la calidad educativa y consecuentemente la satisfacción personal.

Las conclusiones son las siguientes: desarrollo de un sistema de aprendizaje requiere de ambientes, y actividades de organización para el logro de los resultados educativos en los diferentes niveles de formación, el sistema de tutorías permite transferir conocimientos organizados en cursos, módulos de forma presencial o virtual, individual o grupal.

Las tutorías son conceptualizadas desde diferentes formas, sin embargo todas ellas se relacionan con la guía, orientación, colaboración, acompañamiento y asesoría académica, individual o grupal, impartiendo temas seleccionados de asignaturas orientado al desarrollo de la investigación para el aprendizaje.

La tutoría viene asociada a los modelos de tutoría, modelos académicos y modelo educativo.

La tutoría en el proceso de enseñanza - aprendizaje es utilizada de forma individual y/o personalizada, grupal, con ciertas similitudes de aplicación, con relación a que si son obligatorias o voluntarias reguladas por las normas del sistema universitario, siendo en su mayoría de los países estudiados la tutoría como parte del proceso de formación profesional universitario, ya que son obligatorias tanto para el alumno como para el docente.

La tutoría permite lograr beneficios bidimensionales entre el estudiante, el profesor tutor y la institución, ya que se beneficia de la relación tutorial establecida entre el tutor el estudiante y la institución, genera sistemas de acompañamiento colaborativos basados en trayectorias estudiantiles, profesionales y el sistema educativo ya que se obtiene beneficios académicos que pueden ser plasmados en la reducción de las tasas de deserción, repitencia, favorece los procesos de investigación, mejora la calidad de la educación y formación del profesional.

La teoría y la práctica de la tutoría es un ele- mento fundamental para lograr y mejorar la calidad de la educación en las Instituciones de Educación Superior, tal es el caso que se puede llegar a enfatizar que la tutoría desde sus distintas dimensiones y variables aporta a la función de producción educativa y esta a su vez a la función de producción del crecimiento y por ende lograr el desarrollo humano y económico de un país. Por tanto, la tutoría se puede convertir en un mecanismo de calidad para la Educación Superior Universitaria ya que al aplicar en el proceso de enseñanza durante el proceso de formación educativa y titulación reduce la deserción y la repitencia.

\section{AGRADECIMIENTOS}

Agradecemos a la Universidad Técnica de Oruro -Bolivia, Universidad Nacional de Chimborazo - Ecuador por contribuir en el desarrollo y conclusión de la investigación.

\section{REFERENCIAS}

Aguilar M. D. (2013). Correlación existente entre las tutorías y los indicadores de aprobación, retención y eficiencia terminal en la Universidad Autónoma del Estado de Hidalgo. Memoria, sexto encuentro nacional de tutoría, $1-12$.

Alañón R M. T. (2000). La función tutorial. I.C.E. Universidad. Madrid

Almajano P. M. P. $(2000=$. Formación de tutores de. Comunicación presentada en el I Symposium Iberoamericano de Didáctica Universitaria: La calidad de la docencia en la Universidad. Texto disponible en internet .

Álvarez P. P. ., González A. M.C. (2005). La tutoría entre iguales y la orientación universitaria: una experiencia de formación académica y profesional. Revista Educar, 36-107-128.

Álvarez P. P., González A. M.C. (2005). La tutoría académica en la Enseñanza Superior una estrategia docente ante el nuevo reto de la Convergencia Europea. Comunicación presentada la XI Congreso de Formación del Profesorado, Consultado en htpp://aufop. org/xi-congreso/documentos/m4comul. doc.

Álvarez P. P., González A. M.C. (2008). Análisis y valoración conceptual sobre las modalidades de tutoría universitaria en el Espa- 
cio Europeo de Educación Superior. Revista Universitaria de Formación del Profesorado, Universidad de La Laguna, 22 (1), 49-70.

Álvarez P. P., González A. M.C. (2005). La tutoría universitaria en la nueva coyuntura de la enseñanza superior: el programa VELERO. Revista Contextos Educativos, 8-9 y 281-293.

Álvarez P. P., González A. M.C. (2005). El alumnado y la tutoría universitaria un estudios en la Facultad de Educación. Comunicación presentadas al XII Congreso Nacional de Modelos de Investigación Educativa: Investigando en innovación Educativa, Tenerife.

Álvarez P. P. González A. M.C. (2003). Tutoría Universitaria. Servicio de Publicaciones de la ULL. Tenerife.

Álvarez R. V. (1999). Investigación sobre orientación universitaria. Revista de Investigación Educativa, Vol. 17, N², 381 -383, 2 -19.

Anderson J. (2006). Cognitive psychology and its implicatinos (6 ${ }^{a}$. ed.). Worth Publishers. N. New York.

Andrew J. y Hobson P. A. (2009). Corrigendum to "Mentoring beginning teachers: What we know and what we don't". Teach. Teach. Educ. 25, 207-216.

Ariza O. G. Y. (2005). El acompañamiento tutorial como estrategia de la formación personal y profesional: Un estudio basado en la experiencia en una institución de educación superior. Universitas Psychologica, Vol. 4, $N^{\circ} 1,31-41$.

Ariza O. G. I. (2004). El acompañamiento tutoría como estrategia de la formación personal y profesional: un estudio basado en la experiencia en una institución de educación superior. Revista Universidad Psichol, $\mathrm{N}^{\circ} 4$, enero - Junio, 31-41.

Ávila B. H. L. (2006). Introducción a la metodología de la investigación. México : Electrónica, www. eumed.net/libros/2006/203/.

Baptista R. N. O. (2000). Metodología y análisis para evaluar la calidad en la enseñanza superior pública; un caso de estudio. Portugal : Instituto Politécnico de Branganca, Portugal.

Bianculi, K. Y. (2013). Las tutorías universitarias, Estudio de caso: Programa de tutorías PACE$\mathrm{NI}$ de la Facultad de Ciencias Económicas y Sociales de la UNMdP. Universidad. Mar del Plata

Bourdieu P. (1991). El sentido práctico (Trad. Á. Pazos). Madrid: Tauros.

Páginas 60-78
Boud, D. C. (1996). Per Learming and Assessmente. Assessment And Evaluation in Higher Education, University of Tecnology, Sydney, 1-19.

Brown, J. C. (1989). Situated cognition and the culture of learning. Educational Researcher, $18(1), 32.42$.

C.E.U.B. (2008) Comité Ejecutivo de la Universidad Boliviana. (s.f.). Modelo Académico del Sistema de:

C.E.U.B. (2008). Plan de desarrollo Universitaria. 2008 -2013. La Paz: CEUB.

C.E.U.B. (2011). La Universidad Boliviana 2011. La Paz, Bolivia: C.E.U.B. Secretaria Nacional Académica. Bolivia.

Cambours de D. A. M. (2015). La tutoría en la Universidad: una estrategia para la retención de los estudiantes. Conferencia Latinoamericanas sobre abandono en la Educación Superior V CLABES, (págs. 1-8). Talca Chile.

Cano C. C. (2008). Determinantes en la eficiencia en la producción de educación. Revista de la Educación Superior, Vol 37, No 147, Mexico.

Cano G. C. A. (2008). Determinantes en la eficiencia en la producción de educación. Revista de la Educación Superior, vol. XXXVII(3), No 147, julio - Septiembre, , 17-30.

Capelari M. (2009). Las configuraciones del rol del tutor en la Universidad Argentina: aportes para reflexionar acerca de los significados que se construyen sobre el fracaso educativo en la educación superior. Revista Iberoamérica de Educación, n 49/8, 1-10.

Carnicero et. al. (2004). Nuevos retos de la profesión docente. Barcelona: Compendio del II Seminario: Formación, política y gestión universitaria, Universidad de Barcelona.

Carr P. (1999). Alcanzando el futuro: el papel de la mentoria en el nuevo milenio. Peer Resources, British Columbia, Recupaera de www.mentors.ca.

Casanova M. (1992). La Evaluación, Garantía de calidad para el Centro Educativo. EDELVIVES. España.

Castro C. R. A. (2014). Análisis de las buenas prácticas del tutor universitario: estudio de caso en la Universidad Tecnológica de Chihuahua de México. Bellaterra: Tesis Doctoral, Universitat Autónoma de Barcelona.

CEUB. (2014). XII Congreso Nacional de Universidades 2013 - 2014, el Estatuto Orgáni- 
co del Sistema de la Universidad Boliviana . CEUB. La Paz.

Cid S. A. (1998a). La observación y el análisis de las clases de los profesores. Cuaderno de Innovación Educativa, 3, 46-69.

Cid S. et al. (2011). La tutoría en el Practicum. Revista de Educación, 354, Enero - abril, 127-154.

Coriat M. (2005). Orientación y tutoría en la Universidad de Granada. Universidad de Granada. Granada:

Constitución Política del Estado Plurinacional de Bolivia. (2009). La Paz: Ministerio de la Presidencial.

Cuesta C. (2014). Análisis de las buenas prácticas del tutor universitario: estudios de caso en la Universidad de Chihuahua de México. Bellaterra: Universidad Autónoma de Barcelona, Tesis doctoral.

De la Cruz F. G. et al. (2006). Modelo integrador de la tutoría. Revista Mexicana de Investigación Educativa, UNAM, Octubre - Diciembre, Vol. 11, Num.31, 1363-1388.

Dewey, J. (1933). How we think: a restatement of the relation of reflective thinking to the educative process. D. C. Heath. Boston.

Días V. M. (2002). Flexibilidad y educación superior en Colombia: calidad de la Educación Superior. ICFES Bogota.

Doyle, W. (1990). Classroom knowledge as a foundation for teaching. College Record, 91, 347-360.

Echeverría B. (1997). Los servicios universitarios de orientación. EN OPODACA, p\&clobaro (EDS). Calidad en la Universidad: Orientación y Evaluación. Barcelona: LAERTES.

Constitución de la República del Ecuador. Registro oficial 449. 20 de Octubre 2008.

Ley Orgánica de Educación Superior, Registro Oficial Suplemento Nro. 298, 12 de octubre 2010, Quito.

Reglamento General de Estudios Postgrado y Reglamento General de tipos y modalidades de graduación sistema de la Universidad Boliviana. 2002. La Paz: CEUB.

Reglamento del Régimen Académico Estudiantil de la Universidad Boliviana y el modelo académico del Sistema de la Universidad Boliviana. 2011 . La Paz: CEUB.

Reglamento General a la Ley Orgánica de Educación Superior, Decreto Ejecutivo 865 Registro Oficial Suplemento 526 de 02-sep.-
2011

Reglamento del Régimen Académico del Consejo de Educación Superior (CES),2009. ,22 julio 2009.

Edwards A. y Collins J. (1996). Mentoring and developing practice in primary. Buckingham. Oxford University Press.

Elbaz F. (1983). Teacher thinking: A study of practical knowledge. Nichols. New York.

Fensternacher G. (1994). The place of practical arguments in the education of teachers. En V.Richardson (Ed.), Teacher change and the staff development process: A case in reading instruction (pp. 23-42). Teachers College Press. New York.

Ferrer J. (2003). La acción tutorial en la universidad. EN MICAHVILA F Y J-GARCIA DELGADO (Eds.). La tutoría y los modos de aprendizaje en la Universidad. CAM - Cátedra UNESCO. Madrid.

Gairín S. J., M. Feixas., C. Guillamón. y D. Q. Vilamitjana. (2009). La tutoría académica en el escenario europeo de la Educación Superior. Revista interuniversitario de formación de profesorado, $N^{\circ}$ 49, Barcelona, 61-78.

Gallego S. (1997). Las funciones del tutor universitario. Comunicación presentada a la VIII Jornadas Nacionales de la Asociación Española de Orientación y Psicopedagogía. La Orientación Educativa la Intervención Psicopedagógica integradas en el curriculum. Valencia.

García C. J. y A. Seoane. (2007). Tutoría virtual y e-moderación en red. (MONOGRAFICO EN IINEA ). Revista electrónica Teoría de la Educación: Educación y Cultura en la Sociedad de la Información, Vol.8, № 2.

García N. N., I. I. Asensio, R. Carballo, M. García y S. Guardia. (2004). Guía para la labor turorial en la universidad en el Espacio Europeo de la Educación Superior. Programa de Estudios y Análisis de la Dirección General de Universidades. Madrid.

García N. N., I. I. Asensio, R. Carballo, M. García y S. Guardia. (2008). La función tutorial de la universidad en el actual contexto de la Educación Superior. Revista Interuniversitaria de Formación del profesorado, 22(1), 21 48.

García N. N., I. I. Asensio, R. Carballo, M. García y S. Guardia. (2005). La tutoría universitaria ante el proceso de armonización euro- 
pea. Revista de Educación, 189-210.

Greeno, J. C. (1996). Cognition and learning. En D. Berlinery R. Calfee (Eds.), Handbook of educational psychology (pp. 15-46). Simon \& Schuster Macmillan. New York.

Grimmett, P. P. (1992). Craft knowledge and the education of teacher en G. Grant (Ed.), Review of research in education, 18 (pp. 38545). American Educational Research Association. Washington, DC.

Hobson, A. A. (2009). Mentoring beginning teachers: What we know and what we don't. Teaching and Teacher Education, 25, 207216.

Isu, S. y. Perez A. (1998). La tutoría, organización y tareas.. Ed. Graó, Cuarta Edición. Barcelona.

González P. J. 9 de abril de (2015). Las debilidades y retos de las universidades en América Latina. La Prensa, sección sociedad.

Lara G. B. E. (2009). La Tutoría académica en educación superior: modelos, programas y aportes: el caso del Centro Universitario de Ciencias de la Salud. Unidad Editorial del Centro Universitario de Ciencias de la Salud Universidad de Guadalajara. Guadalajara.

Lawler, E. E. (1992). Employee involvement and total quality management. Jossey-Bass.San. Francisco.

Lázaro M. A. J. (2003). Competencias tutoriales en la Universidad. En Michavila, F. y García Delgado, J (Ed). La tutoría y los nuevos modos de aprendizaje en la Feli Arbizu, Clemente Lobato, Laura del Castillo Universidad. Cátedra UNESCO. Madrid .

Lázaro M. A. J. (1986). Manual de Orientación escolar y tutoría. NARCEA. España.

Leinhardt, G. Y. (1995). ntegrating professional knowledge:the theory of practice and the practice of theory. Learning and Instruction," 401-408.

Levinson, D. J. (1978). Seasons of a man's life. Knopf. New York.

Lockheed M. E. y Verspoort. A.M. (1991). Improving primary education in developing countries. Improvving primary education in developing, pag. XIX, 429.

Lopez E. y Oliveros L. (1999). La Tutoría y la Orientación en la Universidad. Revista REOP, 10 (17), 83-98.

Lugo B. (2013). La deserción estudiantil: ¿2Realmente es un problema social? Revista de

Páginas 60-78
Postgrado FACE-UC, Vol. $7 \mathrm{~N}^{\circ}$ 12. Enero -Julio, pp. 289 -309.

Marchesi Á. (2009). Calidad de la Enseñanza en tiempos de cambio. Segunda Edición, Editorial Alianza. España.:

Molina B. I. A. (2012). Estado del arte sobre tutorías. Revista Civilizar. Ciencias Sociales y Humanas, vol.12, num.22, enero - junio, 167-175.

Narro R. J. y Galvan M. A. (2013). La tutoría, un proceso fundamental en la formación de los estudiantes universitarios. Perfiles educativos, Vol. XXXV, Núm. 141, IISUE _ UNAM, 132-151.

Oruro, U. T. (2009). Plan de Desarrollo Estratégico 2009 -2013. Oruro : UTO .

Paravic K. T. y M. Burgos. (2009). Evaluación de calidad de resúmenes de tesis de un programa de magíster en enfermería. . Revista de Ciencia y enfermería XV (3), 55-68.

Isus S. y Pere A. P. (1998). La tutoría, organización y tareas. . Ed. Graó, Cuarta Edición. Barcelona.

Pinilla G. J. (2015). Las debilidades y retos de las universidades en América Latina. La Prensa, sección sociedad.

Ponce J. (2007). Determinantes de la Calidad de la Educación: Una función de Producción Educativa para Ecuador. Tesis de Maestría. FLACSO. Ecuador.

Rodríguez E. S. (2004). Manual de tutoría universitaria, recursos para la acción. Edición Universitaria Octaedro/ICE-UB.

Sogues M. G. (2007). E-tutoría: uso de las tecnologías de la información y comunicación para la tutoría académica universitaria. Revista Electrónica Teoría de la Educación. Educación y cultura en la Sociedad de la Información. Monográfico. Vol. Extraordinario.

Soler M. R. (2003). Mentoring: estrategia de desarrollo de recursos humanos. Ediciones Gestión 2000.Barcelona.

UNESCO. (2006). Informe sobre la Educación Superior en América Latina y el Caribe, 2000 -2005: La metamorfosis de la educación superior. Metropólis C.A. Venezuela.

UNESCO -OIE. (2008). Herramientas de formación para el Desarrollo curricular. SPA , 1-11.

Van Veen D. M. (1997). Los modelos de tutoría: un escenario necesario para la formación del profesorado. Revista interuniversitaria 
formación de profesores, 28, , 119-130.

Wang J. y Odell S. (2002). Mentored learning to teach according to standards based reform: a critical review. Review of Educational Research, 72(3), 481-546.

Wang J. y Odell S. (2002). Mentored learning to teach according to standards-based reform: a critical review. Review of educational research fall, University of Nevada, Las Vegas, 481-546.

Wertsch J. (1991). A sociocultural approach to socially shared cognition. En L. Resnick, J. Levine y S. Teasley (Eds.), Perspectives on socially shared cognition. American Psychological Association. Washington, DC.

Zabalza M. Á. (2003). Competencias docentes del profesorado universitario. Calidad y desarrollo profesional. Ed. Narcea S.A. de Ediciones. Madrid.

Zanz M. (2004). Evaluación de la investigación y sistema de ciencia. Documento de trabajo Consejo Superior de Investigaciones Científicas 04-07. Unidad de Políticas Comparadas, SPRITTE.

Zeichner K. (1993). A formaçao reflexiva de profesores: Ideas e Prácticas. Educa. Lisboa. 